\title{
A Woman With Syncope and Severe, Progressive Headaches
}

\author{
Jennifer Koterwas MS IV, Hind Rahmouni MD, Joanna Kipnes MD
}

\section{Case Report}

A 61 year-old female who was status post-gastrectomy and chemotherapy for gastric cancer presented to the emergency department with complaints of two syncopal episodes and ongoing headaches for the last month. Her syncopal episodes occurred on the day of admission and five days prior and were preceded by shaking, dizziness, and a feeling of being in a "different dimension." The losses of consciousnesses lasted for several minutes and were accompanied by shaking, but were not associated with any urinary or bowel incontinence or tongue biting. The patient denied any fevers or new medications, and felt that the episodes were not associated with a specific activity or time of day. The patient also complained of new onset headaches that had progressed in frequency over the last month from being present for only a few minutes a day to being present for the entire day. The headaches were described as a "brain freeze," with 10 out of 10 pain that was not relieved by acetaminophen with codeine, ibuprofen, or morphine. The headaches were non-radiating and diffusely located throughout the head. The headaches were exacerbated by movement, and associated with an increase in nausea and vomiting. The patient did not have any lacrimation or rhinorrhea, and she denied any past history of migraines, head trauma, or neck pain. Review of systems was negative except for a progressive increase in blurry vision with occasional white spots in her vision over the last three weeks. One week prior to admission she had an outpatient brain MRI (Figure 1a) done, and two days ago a head CT both of which showed no evidence of metastatic disease, hemorrhage, infarct, or mass lesion.

Past medical history was significant for gastric adenocarcinoma (linitus plastica) status-post gastrectomy 3 months ago and neoadjuvant chemotherapy with the last dose 3 weeks ago. The patient was on her fifth week of radiation therapy out of six weeks. Her current medications included as needed acetaminophen with codeine, ibuprofen, prochlorperazine, and lorazepam.

On presentation the patient was afebrile with a temperature of $96.4^{\circ} \mathrm{F}$, and had a respiratory rate of 18 breaths/minute. She had orthostatic heart rate changes as demonstrated by an increase in her heart rate from $87 \mathrm{bpm}$ while supine to $101 \mathrm{bpm}$ while standing. Her supine blood pressure was $160 / 100$ and her standing blood pressure was $177 / 107$. She was fully oriented to person, place, and time. The patient had no nuchal rigidity, nystagmus, or focal neurologic signs. Her cranial nerves, motor, sensory, and cerebellar functions were all intact. Reflexes were $2+$ bilaterally in the upper and lower extremities and gait was normal.

The patient was admitted for a workup of syncope associated with severe, progressive headaches. Intracranial bleeding or a brain tumor was lower on the differential given the recent normal head CT and brain MRI. A cardiac etiology was also unlikely based on the negative history of cardiac disease and a normal physical exam, EKG, and echocardiogram. Dehydration was suspected based on the patient's orthostatic heart rate changes and poor dietary intake. Cerebral vasospasm was thought to be high on the differential for the most likely cause of her headaches also because of unconcerning imaging and unusual presentation.

Seizures appeared to be the most likely cause of her loss of consciousness so an EEG was performed. The EEG revealed focal slow waves greater on the left mid-temporal region than the right and frontal intermittent rhythmic delta activity. Fluid resuscitation was initiated and neurology was consulted to help with diagnosis and treatment. Following the neurologist's recommendation, an MRA of the brain was ordered which revealed no evidence of cerebral artery stenosis or aneurysms.

As there was no improvement in the patient's symptoms, a lumbar puncture was performed on hospital day three which revealed a non-specific pattern of an elevated opening pressure of $24 \mathrm{~mm} \mathrm{H}_{2} \mathrm{O}$, elevated cerebral spinal fluid (CSF) protein of $114 \mathrm{mg} / \mathrm{dL}$, and a normal glucose of $43 \mathrm{mg} / \mathrm{dL}$. On hospital day six the patient became acutely confused, lethargic, and no longer oriented to time. Absence seizures were witnessed by the nurses. Her delirium continued to progress with agitation and

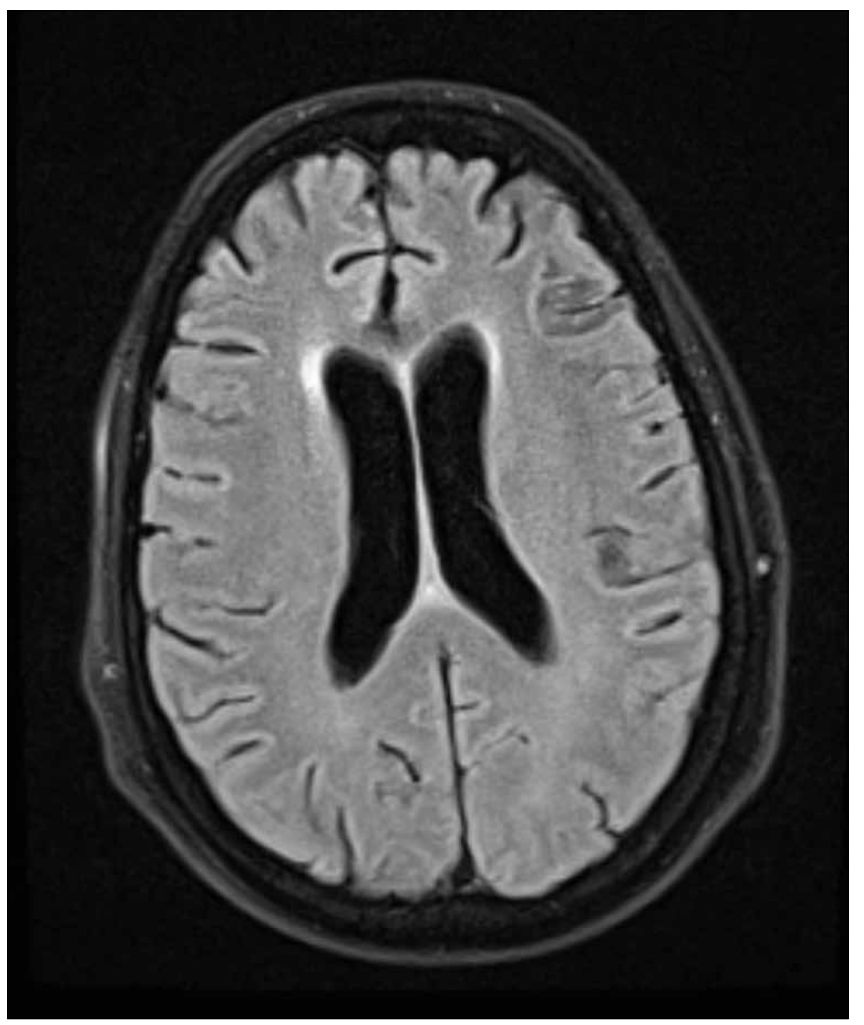

Figure 1a. Initial MRI of the brain showing no evidence of metastatic disease, hemorrhage, infarct, or mass lesion. 
new onset hallucinations and delusions. She also developed an unstable gait, and a new right cranial nerve six palsy. Due to her rapidly declining condition, dexamethasone was started.

Ophthalmology was consulted to examine the patient's visual changes and to evaluate for papilledema due to her elevated opening pressure on the lumbar puncture. On exam, a choroidal lesion was detected that was suspicious for metastasis. The same day, CSF cytology results came back from the initial lumbar puncture revealing atypical cells highly suspicious for cancer of an unknown primary. The results of the lumbar puncture cytology and the suspected metastatic choroidal lesion prompted a repeat MRI and lumbar puncture. The results from the repeat MRI revealed increased FLAIR and enhancement in the subarachnoid space (Figure 1b). The repeat lumbar puncture revealed an elevated opening pressure of 36 $\mathrm{mm} \mathrm{H}_{2} \mathrm{O}$, elevated fluid protein of $93 \mathrm{mg} / \mathrm{dL}$, and a low glucose level of $37 \mathrm{mg} / \mathrm{dL}$, findings which were more consistent with metastatic cancer. The repeat CSF cytology revealed malignant cells consistent with metastatic adenocarcinoma (Figure 2). Both results supported the diagnosis of leptomeningeal carcinomatosis (LC) secondary to linitis plastica adenocarcinoma. The patient was started on corticosteroids.

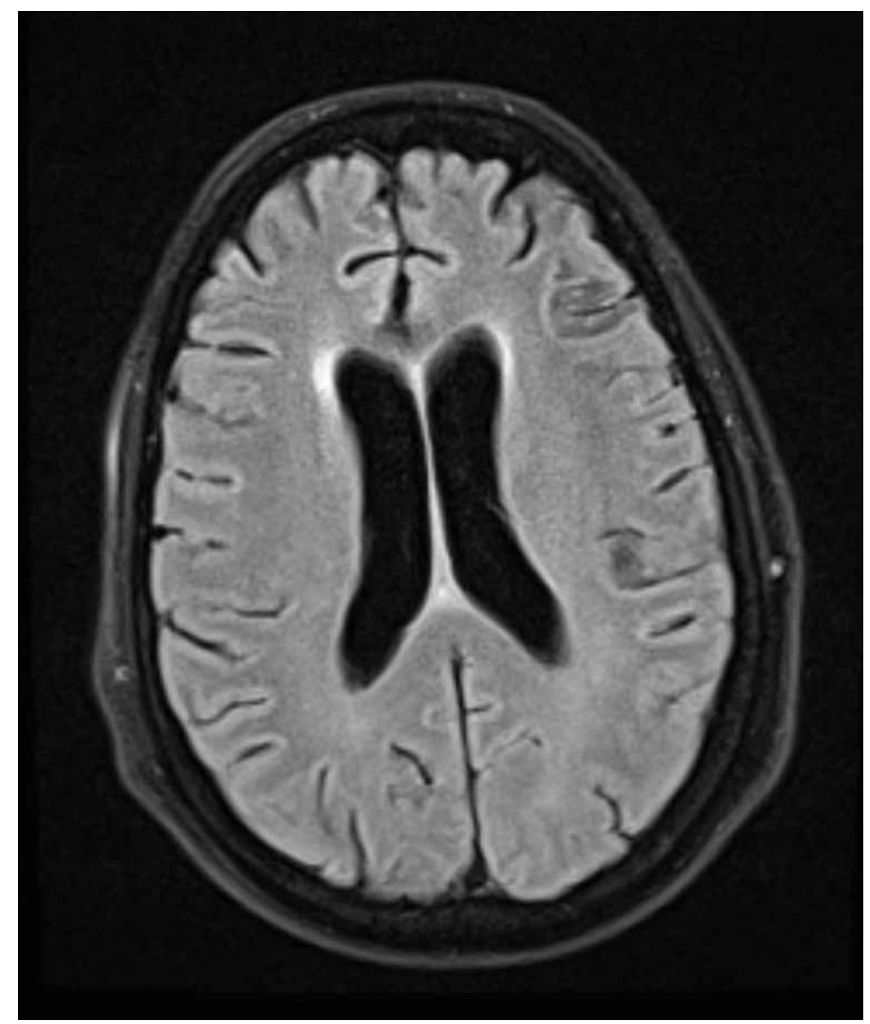

Figure 1b. MRI of the brain, performed two weeks after the first MRI, revealing increased FLAIR signal in the sulci (arrows) and increased enhancement in the subarachnoid space, suggestive of leptomeningeal carcinomatosis.

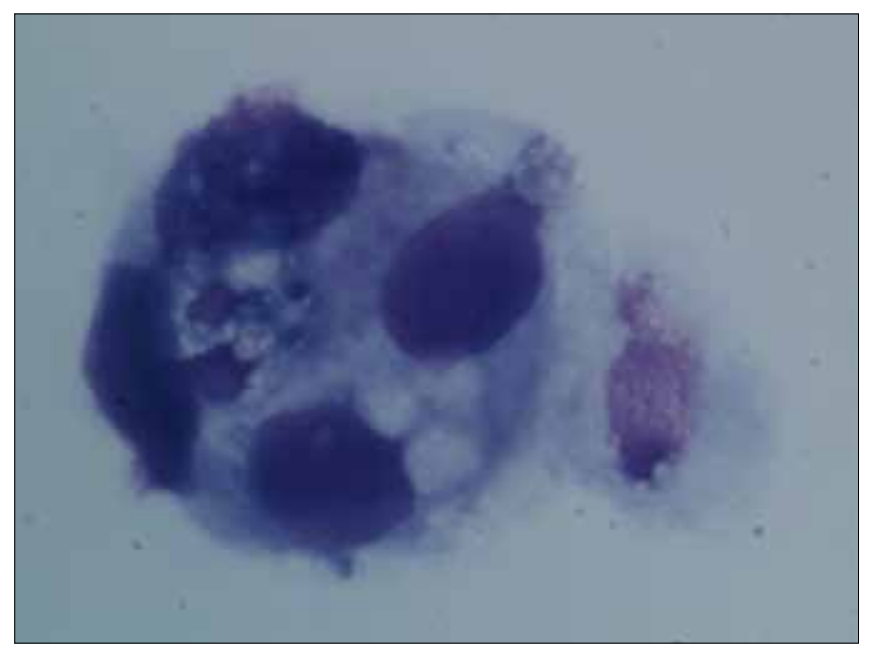

Figure 2. CSF cytology revealing epithelial cells with hyperchromatic and irregularly contoured nuclei, vacuolated cytoplasms, and high nuclear to cytoplasmic ratios. These features are consistent with metastatic adenocarcinoma.

On the ninth day of her hospital course and the third day of steroids, the patient's mental status markedly improved to being fully oriented to person, time, and place with only mild confusion. Her headache also improved and was now present transiently following her steroid treatments. She was able to understand her diagnosis and treatment options, and elected to begin a 5-day course of whole brain radiation followed by intrathecal chemotherapy. Her mental status returned to baseline and she was discharged home on hospital day ${ }^{11}$.

\section{Discussion}

Linitis plastica is a poorly differentiated gastric adenocarcinoma that infiltrates the gastric wall and is commonly associated with peritoneal and lymph node metastases. Histologically, linitis plastica is characterized by signet ring cells along with a marked fibroblastic stroma reaction. ${ }^{3}$ Interestingly, LC arises in only $3 \%$ to $8 \%$ of all solid cancers and most frequently in patients with leukemia, breast cancer, lymphoma, melanoma, and lung cancer. ${ }^{1,3}$ The incidence of LC due to gastric cancer is $0.06 \%$, although the diagnosis may be on the rise due to an increased survival time in gastric cancer patients. ${ }^{2,4}$ Advanced gastric cancer patients classified as Borrmann type III and IV which includes linitis plastica, have higher rates of developing LC when compared to those with early disease. The majority of patients are diagnosed during the treatment of primary gastric cancer as seen in our patient. ${ }^{1,7}$

Extra-abdominal metastasis occurs in less than $13 \%$ of gastric cancer patients with the majority of cases spreading hematogenously to the lung or bone. Although still debated, it is believed that the Batson's venous plexus which consists of longitudinally oriented, valveless paraspinal veins may be the primary route for 
tumor cells to reach the subarachnoid space. ${ }^{1,9}$ Another theory postulates that the gastric cancer spreads by direct extension along peripheral nerves to the subarachnoid space. Once the cancer reaches the leptomeninges and subarachnoid space it has access to all regions of the central nervous system, leading to a variety of nonspecific signs and symptoms. LC's clinical presentations are variable, ranging from isolated to multiple central nervous system, spinal, and cranial nerve symptoms and signs. ${ }^{1}$ Some common clinical symptoms and signs include headaches, nausea, vomiting, altered mental status, seizures, cranial nerve palsie, visual changes, limb weaknesses, and speech difficulties. ${ }^{3,4} \mathrm{LC}$ is difficult to diagnose not only because of the variety of presentations, but also because many of the symptoms resemble common chemotherapeutic induced side-effects, for example nausea, vomiting, sensory loss, hearing loss, vertigo, and nystagmus. ${ }^{1,10}$

Unfortunately there is no single laboratory test or imaging study that is capable of detecting all cases of LC. Diagnosis for LC rests on CSF cytology. The sensitivity of a single lumbar puncture in the detection of atypical cells diagnostic for LC is $54 \%$, but higher sensitivities can be obtained through repeated tests. ${ }^{4}$ The sensitivity of LC increases to $75 \%$ after two lumbar punctures, and up to $85 \%$ after three lumbar punctures. ${ }^{6}$ Other CSF results suggestive of LC include: increased opening CSF pressure, increased CSF cellularity, elevated protein CSF content, and decreased CSF glucose concentration. ${ }^{4}$ The patient described in this case study had an increased CSF pressure, increased protein, and a decreased glucose consistent with the eventual diagnosis of LC.

The imaging study of choice for leptomeningeal carcinomatosis is an MRI of the brain, which characteristically shows focal areas of linear meningeal enhancement in a nodular pattern. The MRI sensitivity for detecting LC however ranges only from $66 \%$ to $77 \%$. Given the low sensitivity of both CSF cytology and MRI when used alone, it has been reported that up to $18 \%$ of patients with LC are likely missed. ${ }^{4}$ The patient described in this case fell into this category because her diagnosis was missed initially based on her first MRI. Contrast enhanced CT scans are also commonly used to evaluate patients but it should be noted that at most the sensitivity is only $44 \%{ }^{4}$

Unfortunately, the prognosis of LC secondary to gastric cancer is poor with a mean survival of four to six weeks for those who do not receive treatment and a mean survival of two to six months for those who receive treatment. ${ }^{1,2,4}$ Some factors that favor survival are a good performance status, a response to intrathecal chemotherapy, a low CSF lactate dehydrogenase concentration, and minimal neurological symptoms. ${ }^{1,7}$ The dismal prognosis of LC in the setting of gastric cancer stresses the importance of early diagnosis because early palliative treatment may help to improve quality of life. A high clinical suspicion is necessary to diagnose LC secondary to gastric cancer due to its nonspecific symptoms, low sensitivity tests, and rare association with gastric cancer. Therefore, even when imaging studies are not supportive as in the above case, the diagnosis of LC should not be ruled out and repeat studies such as a lumbar puncture should be considered. Although only a few cases of intraocular metastasis of gastric adenocarcinoma have been reported, a thorough ophthalmologic exam should also be considered especially in patients with new-onset visual changes as it may reveal metastasis (as in this case) and help to expedite the diagnosis. ${ }^{8}$

\section{References}

1. Lee JL, Kang YK, Kim TW, Chang HM, Lee GW, Ryu MH, Kim E, Oh SJ, Lee JH, Kim SW, Suh C, Lee KH, Lee JS, Kim WK, Kim SH. Leptomeningeal carcinomatosis in gastric cancer. Journal of Neurooncology 66:167-174, 2004

2. Kim MH. Intracranial involvement by metastatic advanced gastric carcinoma. Journal of Neuro-Oncology 43:59-62, 1999

3. Delaunoit T, Boige V, Belloc J, Elias D, Lasser P, Duvillard P, Ducreux M. Gastric linitis adenocarcinoma and carcinomatous meningitis: An infrequent but aggressive association-report of four cases. Annals of Oncology 12:869-871, 2001

4. Lisenko Y, Kumar AJ, Yao J, Ajani J, Ho L. Leptomeningeal carcinomatosis originating from gastric cancer. American Journal of Clinical Oncology 26(2):165-170, 2003

5. DeAngelis, LM. Current diagnosis and treatment of leptomeningeal metastasis. Journal of Neuro-Oncology. 38:245-252, 1998

6. McCrary JA, Patrinely JR, Font RL. Progressive Blindness Caused by Metastatic Occult Signet-ring Cell Gastric Carcinoma. Archives of Ophthalmology. 104(3):410-3, 1986

7. Lee HG, Lee B, Kim SM, Suh BJ, Yu HJ. A Case of Gastric Adenocarcinoma Presenting as Meningeal Carcinomatosis. Korean Journal of Internal Medicine. 22(4):304-7, 2007

8. Sahin A, Kiratli H. Choroidal metastasis as a first sign of recurrence in a patient with gastric adenocarcinoma. Canadian Journal of Ophthalmology. 42(2):331-2, 2007

9. Batson OV. The function of vertebral veins and their role in the spread of metastases. Annals of Surgery. 112:138-45, 1940

10. Wolfgang G, Marcus D, Ulrike S. LC: clinical syndrome in different primaries. Journal of Neuro-Oncology. 38(2-3):103-10, 1998 